\title{
On Electric Fields in Stellarator Equilibria
}

\author{
C.D. Beidler, Y. Igitkhanov, H. Wobig \\ Max-Planck-Institut für Plasmaphysik, Garching bei München, EURATOM-Ass.
}

\begin{abstract}
The paper describes the electric field in stellarator equilibria and discusses the methods how to compute the electric potential. The momentum balance in a given magnetic field including viscous and friction forces is considered in the frame of a multi-fluid model. A general ambipolar condition on closed pressure surfaces is derived which is still valid if magnetic surfaces do not exist. The need for an extended model originates from the singularities of the plasma current in the ideal MHD model of stellarator equilibria, where the parallel current density becomes singular leading to singular parallel electric fields. Viscosity and friction forces eliminate these singularities. The paper investigates the mathematical implications of the extended plasma model and discusses the existence of solutions using the methods of functional analysis.
\end{abstract}

\section{Introduction}

Electric fields in a toroidal equilibrium can be the reason of enhanced radial transport, in particular, when a poloidal $\mathbf{E}$-field exists which leads to a radial $\mathbf{E} \times \mathbf{B}$-drift. In steady state the electric field in stellarators is the gradient of a scalar single-valued potential $\mathbf{E}=-\nabla \Phi$. Plasma resistivity and parallel currents are the reason of a poloidal electric field and the resulting enhanced plasma loss is known as the Pfirsch-Schlüter effect. However, in the frame of ideal equilibrium parallel currents are singular on rational magnetic surfaces, and, as a consequence of Ohm's law $-(\nabla \phi)_{\|}=\eta_{\|}$the parallel electric field also becomes singular.

Often the potential is assumed to be a function of the surface label only. However, this approximation is only valid in regions of good magnetic surfaces and it does not apply to the divertor region, where islands and stochastic regions exist. In neoclassical theory the ambipolar condition is the starting point for computing the radial electric field, however this procedure is only applicable if the potential is a function of a surface label.

In the following we start from the momentum balance of a multi-fluid plasma [1]. In a first approach we neglect the energy balance and restrict the analysis to the momentum balance only. The effect of a temperature gradient will be studied in a separate paper. Furthermore, we restrict the analysis to a collision-dominated plasma, which allows one to use the Braginskii approximation of the viscous forces. In this approximation the viscous stress tensor is locally correlated to the rate of strain tensor, while in neoclassical approximation this relation is non-local. In the second section the basic equations of a multi-species plasma will be described.

\section{The momentum balance}

The momentum balance equations of a multi-fluid model in steady state conditions are

$$
0=-\nabla \cdot \mathbf{S}_{k}+q_{k} n_{k}\left(\mathbf{E}+\mathbf{v}_{k} \times \mathbf{B}\right)+\mathbf{F}_{k}-g_{k} \mathbf{v}_{k} \quad ; \quad k=1, \ldots N
$$


$k$ is the index of the particle species with charge $q_{k}$ and $N$ is the number of particle species. The index $k=N$ denotes the electron equation. $\mathbf{S}_{k}=p_{k}+\pi_{k}+m_{k} n_{k} \mathbf{v}_{k}: \mathbf{v}_{k}$ is the stress tensor of the plasma, $p_{k}$ is the scalar pressure, $\pi_{k}$ the anisotropic component of the pressure tensor and $m_{k} n_{k} \mathbf{v}_{k}: \mathbf{v}_{k}$ is the inertial component of the stress tensor which in case of electrons is small and negligible. The inertial forces of ions may become important in context with a rotating plasma and LH-transition. $\mathbf{F}_{k}=\sum_{i} l_{11}^{k i} \mathbf{v}_{i}, \sum_{k} \mathbf{F}_{k}=0$ is the friction force caused by Coulomb interaction between charged particles [3].

The terms $\nabla \cdot \pi_{k}$ are the viscous forces which in the collisional regime are described by the Braginskii viscosity [2] and in the long-mean free path regime by the neoclassical viscosity. The Braginskii viscosity tensor is a linear function of the rate of strain tensor $W_{\mu v} ; \pi_{i k}=-\sum \eta_{l} W_{l, i k} ; l=0 \ldots 4 . \eta_{l}$ are the viscosity coefficients and $W_{l, i k}$ is related to the rate of strain tensor $W_{\mu v}$.

Close to the plasma boundary charge exchange losses with a neutral background may play a role. This interaction leads to a momentum loss of the ions. For a two-component plasma these friction forces with neutrals have already been formulated by Braginskii ([2], p. 276). We represent this loss by a simple friction term $-g_{k} \mathbf{v}_{k}$. The coefficients $g_{k}>0$ are proportional to the density of neutrals.

Let be $V$ a $N$-dimensional vector space with the elements $\mathbf{v}=\left\{\mathbf{v}_{k}\right\}$ and the scalar product $(\mathbf{v}, \mathbf{u})=$ $\sum_{k}^{N} \mathbf{v}_{k} \cdot \mathbf{u}_{k}$. Next, we define a linear operator $\mathbf{L}$ by $(\mathbf{L v})_{k}=q_{k} n_{k} \mathbf{v}_{k} \times \mathbf{B}+\mathbf{F}_{k}\left(\mathbf{v}_{l}\right)-g_{k} \mathbf{v}_{k}-\nabla \cdot \pi_{k}\left(\mathbf{v}_{k}\right)$ and the non-linear operator $\mathbf{N}$ by $(\mathbf{N v})_{k}:=\mathbf{N}_{k}\left(\mathbf{v}_{k}, \mathbf{v}_{k}\right)=-\nabla \cdot m_{k} n_{k} \mathbf{v}_{k}: \mathbf{v}_{k}$. Furthermore, we define a vector $n \mathbf{v}:=\left\{n_{k} \mathbf{v}_{k}\right\}$ and the force $\mathbf{f}=\left\{\nabla p_{k}+q_{k} n_{k} \nabla \phi\right\}$. The momentum equations and the equations of continuity are now

$$
\mathbf{L v}+\mathbf{N}(\mathbf{v}, \mathbf{v})=\mathbf{f} \quad ; \quad \nabla \cdot n \mathbf{v}=S \quad ; S=\left\{S_{k}\right\}
$$

The net charge produced by the source terms is $\sum_{k} q_{k} S_{k}=0$, which keeps the plasma quasi-neutral. The condition $\sum q_{k} n_{k}=0$ is a condition to the electric potential as will be shown in the following. These equations exhibit a strong similarity to the Navier-Stokes equation of compressible media with $\mathbf{L}$ being the viscosity operator. In a plasma, however, the operator $\mathbf{L}$ also contains friction forces, which couple the various particle species together. A main task of this paper is to show how the techniques of hydrodynamics can be applied to the system (2).

We consider the magnetic field in these equations as given and time independent and focus the attention on the quantities $\mathbf{v}_{k}, p_{k}, \phi$. Pressure and density are correlated by an equation of state. There are $2 N+1$ equations for these $2 N+1$ unknown functions. These equations also describe the steady state plasma in the boundary region of stellarators where nested magnetic islands do not exist and field lines terminate on target plates. The issue of computing the magnetic field self-consistently will be dealt with in a separate paper.

\subsection{The ambipolar condition}

The momentum balance formulated in Eq. (1) also holds in the collisionless limit, however, the viscous tensor must be computed by kinetic equations. In this limit the ambipolar condition often is used to compute the electric potential. This condition results from the sum of all all momentum equations

$$
\nabla p=\mathbf{j} \times \mathbf{B}-\sum_{k} \nabla \cdot\left\{\pi_{k}+m_{k} n_{k} \mathbf{v}_{k}: \mathbf{v}_{k}\right\}-\sum_{k} g_{k} \mathbf{v}_{k}
$$

This procedure eliminates the contribution of the Coulomb friction forces and the electric potential, since $\sum \mathbf{F}_{k}=0$ and $\sum q_{k} n_{k}=0 . p=\sum p_{k}$ is the total plasma pressure. We assume that a plasma equilibrium exists with nested toroidal pressure surfaces $p=$ const.; $|\nabla p| \neq 0$. Existence of magnetic surfaces is not required. We anihilate the $\mathbf{j} \times \mathbf{B}$-term in by multiplying the equilibrium equation (3) with the current density $\mathbf{j}$ and integrate over the pressure surface. Because of $\nabla \cdot \mathbf{j}=0$ and $\nabla \cdot \mathbf{B}=0$ this leads to the relation $<\mathbf{j} \cdot \nabla p>_{p}=0$ and with the help of Eq. (3)

$$
<\mathbf{j} \cdot\left\{\sum_{k} \nabla \cdot\left\{\pi_{k}+m_{k} n_{k} \mathbf{v}_{k}: \mathbf{v}_{k}\right\}+\sum_{k} g_{k} \mathbf{v}_{k}\right\}>_{p}=0 \quad<. .>_{p}=\int_{p} \ldots \frac{d f}{|\nabla s|}
$$


$s$ is the volume of the pressure surface. These equations are valid in all regimes of collisionality and also in rotating plasmas. They show that the damping effect of viscous forces and the friction forces by neutrals must be balanced by the intertial forces. The first equation is the generalized form of the ambipolar condition as will be shown in the following: The current density of each particle species is $\mathbf{j}_{k}=q_{k} n_{k} \mathbf{v}_{k}$ and we define the particle flux $\Gamma_{k}$ across a pressure surface by

$$
q_{k} \Gamma_{k} p^{\prime}(s)=\int_{p} q_{k} n_{k} \mathbf{v}_{k} \cdot \nabla p \frac{d f}{|\nabla s|}=<\mathbf{j}_{k} \cdot \nabla p>_{p}
$$

The sum of these equations is $\sum\left\langle\mathbf{j}_{k} \cdot \nabla p>_{p}=<\mathbf{j} \cdot \nabla p>_{p}=0\right.$, which is equivalent to Eq.(4).

Neglecting the inertial forces and the interaction with neutrals leads to the familiar form of the ambipolar condition $\left\langle\mathbf{j} \cdot \sum_{k} \nabla \cdot\left\{\pi_{k}>_{p}=0\right.\right.$. In the approximation of ideal equilibrium $\nabla p=\mathbf{j} \times \mathbf{B}$ magnetic surfaces and pressure surfaces coincide and the current density in stellarators without toroidal current can be written as $\mathbf{j}=p^{\prime}(s) \mathbf{e}_{p}$, where $\mathbf{e}_{p}$ is the poloidal Hamada base vector. This leads to the ambipolar condition in the form $\left\langle\mathbf{e}_{p} \cdot \sum_{k} \nabla \cdot \pi_{k}>_{p}=0\right.$. This integral relation allows one to compute the potential in the approximation $\phi=\phi(s)$. The tangential component of the electric field cannot be determined. Furthermore, a rotating plasma with Reynolds number larger than unity is also outside the frame of this approximation, since in that case the inertial forces are larger than the viscous forces. If the H-mode transition in fusion experiments is associated with a rotating plasma it is not correct to analyse this phenomenon using the simplified ambipolar condition only.

\section{The Navier-Stokes equations}

The linear operator $\mathbf{L}$ in Eq. (2) is a differential operator of second order; the viscosity operator only occurs in the diagonal elements of the matrix $\mathbf{L}$. We write the operator in the form $\mathbf{L}=\mathbf{L}_{0}+\mathbf{L}_{V}$, where $\mathbf{L}_{0}$ contains all friction forces plus the $\mathbf{v} \times \mathbf{B}$-terms and $\mathbf{L}_{V}$ describes the viscous forces. With respect to the friction forces the plasma differs from the hydrodynamic flow, where only viscous forces occur. The simplest case of a boundary condition is a homogeneous boundary condition $\mathbf{v}=0$, however, due to the finite particle source term this solution is not possible. In order to close the system one needs an equation of state, which correlates pressure to density and temperature.

The appropriate tool to handle Navier-Stokes equations is the search for generalized solutions in Sobolev spaces [4][5]. Let be $H^{m, 2}(\Omega)$ the Sobolev space of order $m$ and $\|\cdot\|_{m}$ the associated norm. $H^{0,2}(\Omega)$ is identical with the Hilbert space $L_{2}(\Omega)$. In the theory of incompressible fluids $H^{1,2}(\Omega)$ is the space used most frequently. Furthermore, the space $H^{1,2}(\Omega)$ is needed, which includes all functions in $\Omega$ with compact support.

\subsection{Constant-density approximation}

In plasmas with cold gas refuelling the density is nearly constant and the pressure gradient is governed by the temperature gradient. In fusion plasmas also small density gradients are expected. Setting the density gradient to zero and introducing the generalized pressure $P_{k}=p_{k}+q_{k} n_{k} \phi, ; n_{k}=$ const leads to

$$
\mathbf{L}_{0} \mathbf{v}+\mathbf{L}_{V} \mathbf{v}+\mathbf{N}(\mathbf{v}, \mathbf{v})=\nabla P \quad ; \quad \nabla \cdot \mathbf{v}=S
$$

The constant densities are included in the source terms $\left(S_{k} / n_{k} \longrightarrow S_{k}\right)$. Taking into account the finite source term, we introduce a velocity $\mathbf{V}_{D}$ by $\nabla \cdot \mathbf{V}_{D}=S$. The ansatz $\mathbf{V}_{D}=-\nabla U+\nabla \times \mathbf{A}$ leads to an elliptic system $-\Delta U=S$, which has a unique solution for any source term $S \in L_{2}(\Omega)$ and the boundary condition $U=0$ on $\partial \Omega$. The boundary condition for the velocity is $-\nabla U+\nabla \times \mathbf{A}=\mathbf{a}$. $\mathbf{a}$ is the velocity on the boundary. There are no other conditions on $\mathbf{A}$, therefore the choice of $\mathbf{A}$ is not unique. If there is no tangential velocity on the boundary we can make the choice $\mathbf{A}=0$. The ansatz for the velocity is now $\mathbf{v}=\mathbf{V}_{D}+\mathbf{w}$ and the resulting equations are

$$
\mathbf{L w}+\mathbf{L} \mathbf{V}_{D}+\mathbf{N}\left(\mathbf{V}_{D}+\mathbf{w}, \mathbf{V}_{D}+\mathbf{w}\right)=\nabla P \quad ; \quad \nabla \cdot \mathbf{w}=0
$$


This system has a remarkable similarity to the Navier-Stokes equation of an incompressible fluid. If we replace the operator $\mathbf{L}$ by $\mu \Delta$ all plasma components are decoupled and the system consists of independent Navier-Stokes equations for every particle species. This suggests to apply the techniques developed in the theory of Navier-Stokes equations [4] also to this system of coupled Navier-Stokes equations of a plasma. As in hydrodynamics one defines the Sobolev space $W(\Omega):=\left\{\mathbf{w} \in H^{1,2}(\Omega) ; \nabla \cdot \mathbf{w}=0\right\}$. Since every vector $\mathbf{v} \in L_{2}(\Omega)$ can be written as the orthogonal sum of a solenoidal vector $\mathbf{a}=\nabla f$ and the incompressible vector $\mathbf{w} ; \nabla \cdot \mathbf{w}=0$, solving the Navier-Stokes equations means to find a vector $\mathbf{w} \in W(\Omega)$, which makes the left hand side of Eq. (7) orthogonal to all $\mathbf{u} \in W(\Omega)$. If such a vector exists, the left hand side is the gradient of functions $P$.

The Stokes problem $\left(\mathbf{L w}+\mathbf{L} \mathbf{V}_{D}=\nabla P\right)$ is the first step and it can be uniquely solved. Since the bilinear form associated with $(\mathbf{u}, \mathbf{L w})_{0}$ is continuous and coercive in $W(\Omega)$ (see appendix), the existence of a unique solution is guaranteed by the Lax-Milgram theorem [6] provided the functional $\left(\mathbf{u}, \mathbf{L} \mathbf{V}_{D}\right)_{0}$ is bounded in $W(\Omega)$. This is the case, since $\left\|\mathbf{V}_{D}\right\|_{1}$ depends only on the source term $S$ and the boundary conditions and therefore has an upper limit.

A generalized solution $\mathbf{w} \in W(\Omega)$ exists, if the left hand side in equation (7) is orthogonal in $L_{2}$ to all $\mathbf{u} \in W(\Omega)$. In order to check this we consider all functionals separately. The first one is $(\mathbf{u}, \mathbf{L w})_{0}$ which is a bounded functional uf $\mathbf{u}$ with fixed $\mathbf{w}$. According to the theorem of Riesz there exists a bounded operator $\mathbf{Z}$ with $(\mathbf{u}, \mathbf{L w})_{0}=(\mathbf{u}, \mathbf{Z w})_{1}$. The inverse $\mathbf{Z}^{-1}$ exists and is bounded in $W(\boldsymbol{\Omega})$.

The second functional associated with the left hand side is $F(\mathbf{u})=\left(\mathbf{u}, \mathbf{L} \mathbf{V}_{D}+\mathbf{N}\left(\mathbf{V}_{D}+\mathbf{w}, \mathbf{V}_{D}+\mathbf{w}\right)\right.$, which is also bounded in $W(\Omega)$. The first part on the right hand side yields $\left(\mathbf{u}, \mathbf{L} \mathbf{V}_{D}\right)_{0}=\left(\mathbf{u}, \mathbf{Z} \mathbf{V}_{D}\right)_{1}$. The second part of the functional already occurs in the hydrodynamic theory of incompressible flows [5][4], where the boundedness has been proved. This implies that a non-linear operator $\mathbf{K}$ exists, so that the relation holds $\left(\mathbf{u}, \mathbf{N}\left(\mathbf{V}_{D}+\mathbf{w}, \mathbf{V}_{D}+\mathbf{w}\right)\right)_{0}=(\mathbf{u}, \mathbf{K w})_{1}$ The system of Navier-Stokes equations is equivalent to the operator equation

$$
\mathbf{Z w}+\mathbf{K w}=-\mathbf{Z} \mathbf{V}_{D}
$$

The operator $\mathbf{Z}$ contains all plasma properties while the operator $\mathbf{K}$ is associated with inertial terms only and the vector $\mathbf{V}_{D}$. In hydrodynamic theory the compactness of the operator $\mathbf{K}$ has been shown, which allows one to refer to the Leray-Schauder fixed point theorem [7]. The proof that a fixed point exists follows the same line as in hydrodynamic theory. Furthermore, all solutions are bounded in $W(\Omega)$ with a bound, which depends on $\mathbf{V}_{D}$ and the properties of the operator $\mathbf{Z}$. This implies that also the plasma current density $\sum q_{k} n_{k} \mathbf{v}_{k}$ is bounded. In contrast to the Stokes equations the solutions of the Navier Stokes model are not unique, several solutions and bifurcations can arise. It needs to be investigated when the solutions are unique. In analogy to the hydrodynamic case it is expected that the solution $\mathbf{w}$ is unique if the friction forces and the viscous forces are large enough and the velocity $\mathbf{V}_{D}$ is small enough.

In contrast to a hydrodynamic flow, where external forces or inflow and outflow through the boundary maintain the flow against viscous dissipation, the plasma has no external forces, which for example force the plasma to rotate. Instead, the role of an external force is played by the source-induced velocity $\mathbf{V}_{D}$. This enters the momentum balance through $\mathbf{L} \mathbf{V}_{D}$ and $\mathbf{N}\left(\mathbf{V}_{D}, \mathbf{V}_{D}\right)$. However, the most interesting term is the coupling term between the radial flow $\mathbf{V}_{D}$ and the convective flow $\mathbf{w}$ in the inertial forces $\mathbf{N}\left(\mathbf{V}_{D}, \mathbf{w}\right)+\mathbf{N}\left(\mathbf{w}, \mathbf{V}_{D}\right)$. In the Stringer spin-up mechanism this coupling is the reason for plasma rotation.

\subsection{Barotropic approximation}

In this approximation the pressure is an increasing function of the density only: $p_{k}=p_{k}\left(n_{k}\right) ; p^{\prime}>0$. Using this approximation and $n_{k}>0$ we introduce a new function $u_{k}$ by

$$
u_{k}:=F_{k}\left(n_{k}\right)=\int_{N_{k, 0}}^{n_{k}} \frac{p_{k}^{\prime}(x) d x}{x} \quad ; \quad \nabla u_{k}=\frac{\nabla p_{k}}{n_{k}}
$$

This function is uniquely invertible yielding $n_{k}=G_{k}\left(u_{k}\right) ; G^{\prime}>0$. Next, we introduce the generalized pressure $P_{k}=u_{k}+q_{k} \phi$ and utilize the condition of quasi-neutrality in order to compute the electric 
potential in terms of the generalized pressure. This condition is

$$
\sum_{k} q_{k} n_{k}=0 \longrightarrow \sum_{k} q_{k} G_{k}\left(P_{k}-q_{k} \phi\right)=0 \longrightarrow \phi=\phi\left(P_{1}, \ldots, P_{N}\right), n_{k}=n_{k}\left(P_{1}, \ldots, P_{N}\right)
$$

The potential is a function of the generalized pressure. Inserting this result into $u_{k}$ makes the density a function of the generalized pressure and the basic equations are

$$
\mathbf{L}_{0} \mathbf{v}+\mathbf{L}_{V} \mathbf{v}+\mathbf{N}(\mathbf{v}, \mathbf{v})=n \nabla P \quad ; \quad \nabla \cdot n \mathbf{v}=S
$$

The electricc potential has been eliminated and the unknown functions are $\mathbf{y}_{k}, P_{k}$. The importance of the source term is demonstrated by $S=0$ and the assumption that a solution $\mathbf{w} \in H^{1,2}(\Omega)$ exists. This leads to $(\mathbf{w}, n \nabla \psi)_{0}=0$ and $\left(\mathbf{w}, \mathbf{N}(\mathbf{w}, \mathbf{w})_{0}=0\right.$. The momentum balance is then $(\mathbf{w}, \mathbf{L w})_{0}=0$, which implies $\mathbf{w}=0$. In this case no finite solution exists.

Since the friction forces (including the $\mathbf{v} \times \mathbf{B}$-terms) are the larges ones, one is tempted to use the solution of the friction model as a lowest order approximation. We introduce the velocity $\mathbf{V}_{D}$ by $\mathbf{L}_{0} \mathbf{V}_{D}=$ $n \nabla P$ and set $\mathbf{v}=\mathbf{V}_{D}+\mathbf{w}$ with $\mathbf{w} \in H^{1,2}(\Omega)$. The resulting equations are

$$
\mathbf{L} \mathbf{w}+\mathbf{L}_{V} \mathbf{V}_{D}+\mathbf{N}\left(\mathbf{V}_{D}+\mathbf{w}, \mathbf{V}_{D}+\mathbf{w}\right)=0 \quad ; \quad-\nabla \cdot n \mathbf{L}_{0}^{-1} n \nabla P+\nabla \cdot n \mathbf{w}=S
$$

$\mathbf{L}_{0}^{-1}$ is the inverse matrix of $\mathbf{L}_{0}$. The first equation has the same structure as in the constant-density model; given the velocity $\mathbf{V}_{D}$ the convective velocity $\mathbf{w}$ can be computed from the momentum balance. Given the convective velocity, the functions $P_{k}$ follows from the equations of continuity, which are a second-order system for the $P$-functions. However, the highest derivatives of $P_{k}$ occur in $\mathbf{L}_{V} \mathbf{V}_{D}$ and therefore the convergence of the iterative procedure is an open question. If the existence of a solution and the boundedness of $\left\|\mathbf{V}_{D}\right\|_{1}$ can be proved by a different method, these equations (12) can be used to find approximations.

Since in the barotropic model the plasma is compressible, the theory of compressible fluids may provide some guide lines how to prove the existence of solutions. In a compressible fluid the viscous operator is $\mathbf{L}_{V}=\mu \Delta+v \nabla \nabla \cdot$. ; a friction term and the electric field do not occur. An existence theorem for compresible fluids with external force and a particle source term has been established by Beirão de Veiga [8]. M. Padula [9] has also proved an existence and uniqueness theorem for compressible fluids without volume source.

\subsection{Ideal gas approximation}

In order to avoid the analysis of the coupled system of energy equations and momentum balance, we consider the temperature as given function $T_{k}(\mathbf{x})>0$ in the momentum balance and assume that pressure and temperature are linked via the ideal gas law $p_{k}=n_{k} T_{k}$. $T_{k}$ stands for $K T_{k}$, where $K$ is the Boltzmann constant. Next we introduce a generalized pressure by $P_{k}=T_{k} \ln p_{k}+q_{k} \phi$, which allows one to write the right hand side of the momentum equation as

$$
\nabla p_{k}+q_{k} n_{k} \phi=n_{k} \nabla P_{k}-p_{k} \ln p_{k} \nabla \ln T_{k}
$$

The electric potential results from the condition of quasi-neutrality, which defines the electric potential implicitly

$$
H=\sum_{k} \frac{q_{k}}{T_{k}} \exp \left[\frac{1}{T_{k}}\left(P_{k}-q_{k} \phi\right)\right]=0 \longrightarrow \phi=\phi\left(P_{l}, T_{l}\right)
$$

The derivative of $H$ with respect to $\phi$ is negative and therefore the relation is invertible yielding the electric potential is a continuous function of the generalized pressure and the temperature. Inserting this into the density gives $n_{k}=n_{k}\left(P_{l}, T_{l}\right)$. The momentum balance is now

$$
\mathbf{L}_{0} \mathbf{v}+\mathbf{L}_{V} \mathbf{v}+\mathbf{N}(\mathbf{v}, \mathbf{v})=n \nabla P-\mathbf{F} \quad ; \quad \nabla \cdot n \mathbf{v}=S
$$


The external force $\mathbf{F}$ is $\mathbf{F}=\left\{F_{k}=p_{k} \ln p_{k} \nabla \ln T_{k}\right\}$. We get the same equations as in the barotropic model exept for the additional external force.

In summary we have shown that under mild assumptions on the equation of state the electric potential can be eliminated from the momentum balance equations using the condition of quasi-neutrality. The unknown functions in the momentum equations and the continuity equations are the plasma velocities and the generalized pressures. The electric potential is a unique function of the generalized pressures and the temperatures. The tools of compressible and incompressible fluid theory can also be applied to the multi-fluid model of a quasi-neutral plasma. It has been shown that in rotating plasmas with Reynolds number greater than unity the ambipolar condition is strongly affected by inertial forces.

\section{Appendix: The operator $L$}

In the following we will show that the bilinear functional $F(\mathbf{u}, \mathbf{v})=-(\mathbf{u}, \mathbf{L v})$ is coercive and continuous in $H^{1,2}(\Omega)$. For this purpose we consider the scalar product

$$
-(\mathbf{u}, \mathbf{L v})_{0}=-\sum_{k} \int_{\Omega} \mathbf{u}_{k} \cdot \mathbf{L}_{k} \mathbf{v}_{k} d^{3} \mathbf{x} \quad ; \quad \mathbf{u}, \mathbf{v} \in \stackrel{\circ}{H}^{1}(\Omega)
$$

First, let us prove coerciveness of this functional. Setting $\mathbf{u}=\mathbf{v}$ we obtain after some algebra

$$
-(\mathbf{v}, \mathbf{L v})_{0}=\frac{1}{2} \int_{\Omega} \sum_{k i} l_{11}^{i k}\left(\mathbf{v}_{k}-\mathbf{v}_{i}\right)^{2} d^{3} \mathbf{x}+\int_{\Omega} \sum_{k} g_{k} \mathbf{v}_{k}^{2} d^{3} \mathbf{x}+\int_{\Omega} \sum_{k} \mathbf{v}_{k} \cdot \nabla \cdot \pi_{k} d^{3} \mathbf{x} \geq 0
$$

The first term on the right hand side is the work on the friction forces, the second one is the work by plasma neutral interaction and the last one is the work dissipated by viscous forces. All terms are positive except for $\mathbf{v}=0$. The eigenvalues of the matrix $-l_{11}^{i k}+g_{k} \delta_{i k}$ are positive and non-zero. Let $\lambda_{0}>0$ be the lowest eigenvalue in $\Omega$. This leads to the estimate

$$
\frac{1}{2} \int_{\Omega} \sum_{k i} l_{11}^{k i}\left(\mathbf{v}_{k}-\mathbf{v}_{i}\right)^{2} d^{3} \mathbf{x}+\int_{\Omega} \sum_{k} g_{k} \mathbf{v}_{k}^{2} d^{3} \mathbf{x} \geq \lambda_{0} \int_{\Omega} \sum_{k} \mathbf{v}_{k}^{2} d^{3} \mathbf{x}=\lambda_{0}\|\mathbf{v}\|_{0}^{2}
$$

The work dissipated by viscous forces is also positive, which, after some lengthy but straightforward manipulations using the properties of the Braginskii viscosity, leads to the estimate

$$
\int_{\Omega} \mathbf{v} \cdot \nabla \cdot \pi d^{3} \mathbf{x} \geq \frac{2}{3} \eta_{\min } \int_{\Omega}\left\{\left(\frac{\partial v_{i}}{\partial x_{k}}\right)^{2}+\frac{1}{3}(\nabla \cdot \mathbf{v})^{2}\right\} d^{3} \mathbf{x} \geq \frac{2}{3} \eta_{\min }\|\mathbf{v}\|_{1}^{2}
$$

$\eta_{\min }$ is the smallest of the viscosity coefficients. Combining the results leads to $-\left(\mathbf{v}, \mathbf{L v} b_{0} \geq c_{0}\|\mathbf{v}\|_{1} ; c_{0}>\right.$ 0 . In a similar manner one can show that $\left|(\mathbf{u}, \mathbf{L v})_{0}\right| \leq C_{0}\|\mathbf{u}\|_{1}\|\mathbf{v}\|_{1}$. These are the conditions of the LaxMilgram theorem, which says that the equation $\mathbf{L v}=\mathbf{g}$ has a weak solution, which means that there exists a unique element $\mathbf{v} \in \stackrel{\circ}{H}^{1,2}$ satisfying the condition $(\mathbf{u}, \mathbf{L v})_{0}=(\mathbf{u}, \mathbf{g})_{0}$ for all $\mathbf{u} \in \stackrel{\leftrightarrow}{H}^{1,2}(\Omega)$.

\section{References}

[1] H. Wobig, Plasma Phys. Control. Fusion 38 (1996) 1053

[2] S.I. Braginskii, Rev. of Plasma Phys. Vol. I, p. 250, Consultants Bureau New York 1965

[3] S. Hirshman, D. Sigmar, Nuclear Fusion 21, (1981) p.1080-1198

[4] Temam R, Navier-Stokes Equations, North Holland 1977

[5] O.A. Ladyshenskaja, Funktionalanalytische Untersuchungen der Navier-Stokesschen Gleichungen, Akademie-Verlag, Berlin 1965

[6] D. Gilbarg, N.S. Trudinger, Elliptic Partial Differential Equations of Second Order p. 83, Springer-Verlag 1983

[7] D. Gilbarg, N.S. Trudinger, Elliptic Partial Differential Equations of Second Order p. 279, Springer-Verlag 1983

[8] H. Beirão da Veiga, Comm. Math. Phys. 109, 229-248, (1987)

[9] M. Padula , Arch. Rational Mech. Anal. 77, 89-102, (1987) 